\title{
Characterization of Resistance to Major Tropical Root-Knot Nematodes (Meloidogyne spp.) in Solanum sisymbriifolium
}

\author{
Abolfazl Hajihassani, ${ }^{1, \dagger}$ William B. Rutter, ${ }^{2}$ Tanner Schwarz, ${ }^{3}$ Moges Woldemeskel, ${ }^{4}$ Md Emran Ali, ${ }^{1}$ and Negin Hamidi ${ }^{1}$ \\ ${ }^{1}$ Department of Plant Pathology, University of Georgia, Tifton, GA 31793, U.S.A. \\ 2 United States Department of Agriculture-Agricultural Research Service United States Vegetable Laboratory, Charleston, SC 29414, U.S.A. \\ ${ }^{3}$ Department of Entomology and Plant Pathology, North Carolina State University, Raleigh, NC 27695, U.S.A. \\ ${ }^{4}$ Department of Pathology, University of Georgia, Tifton, GA 31793, U.S.A. \\ Accepted for publication 11 December 2019.
}

\begin{abstract}
Root-knot nematodes (Meloidogyne spp.) are important contributors to yield reduction in tomato. Though resistant cultivars to common species (Meloidogyne arenaria, M. incognita, and M. javanica) are available, they are not effective against other major species of rootknot nematodes. Cultivars or lines of Solanum sisymbriifolium were examined to assess the presence and level of resistance to five major species: $M$. arenaria race $1, M$. incognita race 3, $M$. haplanaria, M. javanica, and M. enterolobii. Differences in S. sisymbriifolium response to the nematode infection were apparent when susceptibility or resistance was classified by the egg counts per gram fresh weight of root and the multiplication rate of the nematodes. The cultivar Diamond was highly

increased 2.5-fold from the initial population of the M. incognita on Diamond. All S. sisymbriifolium cultivars were highly resistant to both $M$. haplanaria and $M$. enterolobii, while highly susceptible to $M$. javanica. A microplot study under field conditions using Sis Syn II confirmed that $M$. arenaria, $M$. incognita, and $M$. haplanaria were not pathogenic on the plant. Likewise, an examination on cross-sections of galled root tissues confirmed the susceptibility and resistance of S. sisymbriifolium lines to Meloidogyne spp. Using S. sisymbriifolium as a resistant rootstock or a new source of resistance may result in the development of nonchemical and sustainable management strategies to protect the tomato crop.
\end{abstract} susceptible, Quattro and White Star were susceptible, while Sis Syn II was resistant to M. arenaria. Quattro, White Star, and Sis Syn II exhibited a moderate to high level of resistance to $M$. incognita but the nematode
Keywords: histophatology, nematology, resistance, root-knot nematode, Solanum sisymbriifolium, tomato
Tomato (Solanum lycopersicum Mill.) is one of the major vegetable crops cultivated and consumed internationally. In the United States, tomato is the most extensively grown vegetable as a fullseason summer or fall crop. In 2018, the fresh market and processing tomato produced in the country were valued at $\$ 1.86$ billion (USDA-NASS 2019). Tomato is often exposed to attack by root-knot nematodes (Meloidogyne spp.), the most aggressive and widely distributed nematode pest across a wide range of climates (Hussey and Janssen 2002; Sasser et al. 1983; Wesemael et al. 2011). Meloidogyne-infected plants show abnormal growth of the root systems, characterized by galls which limit the uptake of nutrients and water and inhibit mineral translocation. This will result in wilting, stunted growth, and, consequently, severe yield losses (Abad et al. 2003; Hussey 1985). Though it has been estimated that root-knot nematode numbers as low as 4 juveniles per $100 \mathrm{~cm}^{3}$ of soil can cause significant yield loss-up to $85 \%$ on tomato in sandy-textured soils (Baker et al. 1976) - the damage threshold of Meloidogyne spp. on vegetable crops is only 1 nematode per $100 \mathrm{~cm}^{3}$ of soil. The three most prevalent species reported to attack tomato in the United States are Meloidogyne incognita, M. arenaria, and M. javanica. Each of these species can cause severe yield reduction and quality damage (Baker et al. 1976). In recent years, three other species-M. floridensis (Church 2005), M. haplanaria (Joseph et al. 2016), and M. enterolobii (Brito et al. 2004; Rutter et al. 2019)—were detected with the capability of infecting and damaging vegetable crops including tomato (Kiewnick et al. 2008).

†Corresponding author: A. Hajihassani; abolfazl.hajihassani@uga.edu

The author(s) declare no conflict of interest.

(C) 2020 The American Phytopathological Society
In the United States, chemical control is the most predominant tactic for managing nematodes in intensive cultivation systems of vegetables. Soil fumigants such as 1,3-dichloropropene and chloropicrin or their mixture (i.e., Pic-Clor 60 or Telone C-35), as well as nonfumigant nematicides, are used to control root-knot nematodes in vegetables, including tomato systems (Hajihassani 2018; Hajihassani et al. 2019a). However, the application of nematicides is often associated with increased production costs and they have a short-term efficacy in the soil, with root-knot nematode populations increasing at the end of the growing season (Hajihassani et al. 2019b). The most effective, environmentally friendly, and economical means of controlling Meloidogyne spp. is the use of resistant cultivars. Use of resistance has recently increased because of increasing environmental concerns and regulatory restrictions on the application of chemical products in conventional systems.

Many wild Solanum spp. display resistance to Meloidogyne spp.; however, the resistance is often not complete and nematodes may form a few galls or eggs. An introgression from Lycopersicon peruvianum into cultivated tomato (Bailey 1941; Gilbert and McGuire 1956) provided the Meloidogyne-resistant gene 1 (Mi-1), which confers resistance to three major species: $M$. javanica, $M$. incognita, and $M$. arenaria (Williamson 1998). The $M i-1$ gene has evolved as an economically sustainable and effective approach for controlling Meloidogyne spp. However, the spread of species that overcome plant resistance such as $M$. enterolobii and M. haplanaria (Brito et al. 2004; Kiewnick et al. 2008) and virulent populations (biotypes) of Meloidogyne spp. (Castagnone-Sereno et al. 1994; Iberkleid et al. 2014; Ornat et al. 2001; Tzortzakakis et al. 2005) have reduced the efficacy of $M i-1$-mediated resistance.

In this scenario, root-knot nematode management should involve the search for new resistant genes or rootstocks and the development of sustainable strategies aimed at reducing the nematode population density below the damage threshold to prevent economic losses. 
S. sisymbriifolium, known as "litchi" tomato or "sticky nightshade", is a solanaceous annual or perennial plant species that has been introduced to the United States from South America and is known to occur in many states in the country (Kartesz 2012). The plant contains a toxic substance, solasodine, which helps make the plant resistant to some pests and diseases. S. sisymbriifolium has been examined for its efficacy in reducing the development and reproduction of root-knot, cyst (Globodera spp.), and root-lesion (Pratylenchus spp.) nematodes (Dias et al. 2012; Scholte and Vos 2000; Timmermans et al. 2006). It has been used as a resistant rootstock for controlling $M$. incognita in tomato production (Baidya et al. 2017; Matsuzoe et al. 1993). This work describes the host status of $S$. sisymbriifolium for resistance to five major root-knot nematode species in a controlled greenhouse.

The durability of crop resistance to root-knot nematodes depends on some biotic and abiotic factors. A field study will show whether the potential resistance in $S$. sisymbriifolium selected in the greenhouse is effective under field conditions, in which the test crops may be subjected to higher numbers of the nematode for an extended period of time. Furthermore, the temperature is an important factor for invasion, development, and reproduction of root-knot nematodes. In tomato, however, $M i-1$ gene resistance is overcome by heat stress. Although some disagreements are present in the literature, resistance has been reported to be inactive in soil temperatures above $28^{\circ} \mathrm{C}$ (Abdul-Baki et al. 1996; de Carvalho et al. 2015; Dropkin 1969). Therefore, the $S$. sisymbriifolium response to root-knot nematode infestation was also investigated in microplots under field conditions for comparison of the results with those from glasshouse experiments. Our overall goal was to characterize S. sisymbriifolium cultivars with high levels of resistance for use as a rootstock or a new source of genetic resistance for Meloidogyne control in tomato.

\section{MATERIALS AND METHODS}

Plant source. Seed of $S$. sisymbriifolium cultivars White Star, Diamond, and Quattro were obtained from P. H. Petersen, Lundsgaard, Germany. The S. sisymbriifolium line Sis Syn II was obtained from C. Brown (United States Department of AgricultureAgricultural Research Service, Prosser, WA, U.S.A.). Tomato (cultivar Rutgers) was used as the susceptible control.

Nematode culture. All Meloidogyne spp. used in this study were specifically identified using species-specific primers and mitochondrial genes (Marquez et al. 2019; Rutter et al. 2019). Isolates of $M$. incognita race $3, M$. arenaria race $1, M$. javanica, and $M$. haplanaria were isolated from different crops and increased separately on tomato (cultivar Rutgers) or eggplant (cultivar Black Beauty) pot cultures in the greenhouse for 10 to 12 weeks. To prepare inoculum, second-stage juveniles (J2) of each species were harvested from galled roots by incubating chopped roots in a mist chamber for 7 days. The juveniles collected from a 38- $\mu \mathrm{m}$-aperture sieve were washed and resuspended with tap water at 1,000 J2/ml.

Two isolates of $M$. enterolobii from North Carolina (NC1 and NCSP) and one isolate from South Carolina (BASP) were isolated from sweet potato and cultured on tomato (Rutgers). Eggs were extracted from infected roots with a $1 \%$ sodium hypochlorite technique (Hussey and Barker 1973). Eggs collected from a 25- $\mu \mathrm{m}-$ aperture sieve were counted under an inverted microscope and resuspended at 10,000 eggs $/ \mathrm{ml}$. The nematode inocula were stored at $4^{\circ} \mathrm{C}$ up to 1 week until use.

Reproduction of $M$. arenaria, $M$. incognita, $M$. haplanaria, and $M$. javanica in the greenhouse. Two independent experiments for each nematode species were conducted in 2018 in the greenhouse at $26 \pm 2{ }^{\circ} \mathrm{C}$. Seed of S. sisymbriifolium and tomato were planted in Miracle-Gro Moisture Control potting mix (The Scotts Miracle-Gro Company, Marysville, OH, U.S.A.) in seed trays (Speedling Incorporated, Ruskin, FL, U.S.A.). When seedlings were 2 to 3 weeks old and approximately 5 to $7 \mathrm{~cm}$ tall, they were transplanted individually into Deepot D40L cells $(6.9 \mathrm{~cm}$ in diameter by $25.4 \mathrm{~cm}$ deep; Stuewe \& Sons, Inc., Tangent, OR, U.S.A.) containing equal parts ( vol/vol) of steamed field soil and washed sand. After 2 days, potted soils were watered and a suspension of 1,000 J2 each of $M$. arenaria, $M$. incognita, $M$. haplanaria, or $M$. javanica was pipetted into two 4-cm-deep holes in the soil near the root ball. Rutgers tomato was included as a susceptible control. The plants were placed on support trays (Stuewe \& Sons, Inc.) in a completely

TABLE 1. Reproduction of Meloidogyne arenaria race 1, M. incognita race 3, M. haplanaria, and M. javanica on Solanum sisymbriifolium in the greenhousew

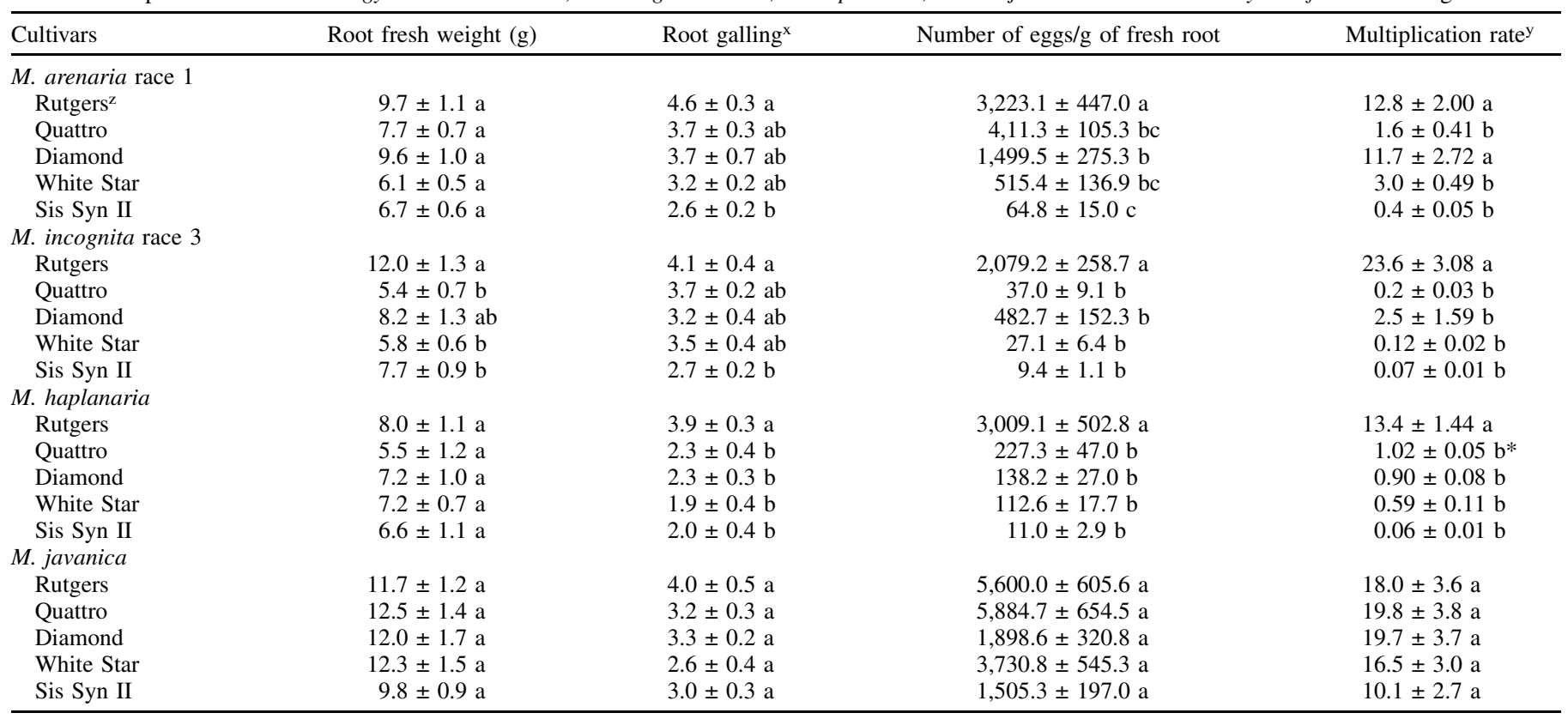

w Each value signifies the mean \pm standard error $(n=10)$ of each treatment. Means followed by the same letter within columns are not significantly different $(P=0.05)$ based on Tukey's test. The asterisk shows that the multiplication rate is not different from 1 according to least-squares means $t$ tests.

${ }^{x}$ Rated on a scale of 0 to 5 , where $0=$ no gall, $1=1$ to 2 galls, $2=3$ to 10 galls, $3=11$ to 30 galls, $4=31$ to 100 galls, and $5=>100$ galls on the root system.

y Multiplication rate $=$ the number of eggs on roots at harvest/inoculated juveniles at planting.

${ }^{\mathrm{z}}$ Rutgers $=$ susceptible tomato control. Each plant was inoculated with 1,000 second-stage juveniles of each nematode species at transplanting. 
randomized design, with five replicates for each cultivar and nematode species. Plants were watered once a day with equal amounts of water, and fertilized once during the experiment with $2 \mathrm{~g}$ of Osmocote smart-release fertilizer (15-9-12; The Scotts Miracle-Gro Company).

Experiments were terminated 8 weeks after nematode inoculation, at which point the plants were removed from the pots and their root systems were gently washed free of soil with running water, air dried briefly on paper towels, and weighed. The root systems infected with $M$. incognita, $M$. arenaria, $M$. javanica, and $M$. haplanaria were visually scored for gall severity using a scale of 0 to 5 , where $0=$ no gall, $1=1$ to 2 galls, $2=3$ to 10 galls, $3=11$ to 30 galls, $4=31$ to 100 galls, and $5=>100$ galls on the root system (Taylor and Sasser 1978). Following gall severity assessment, all nematode eggs were recovered from roots (Hussey and Barker 1973) and counted using an inverted microscope, and the numbers of eggs per gram of root were determined. Also, the multiplication rate of the nematodes was determined by dividing the final egg count in roots at harvest $(\mathrm{Pf})$ by the initial inoculum density $(\mathrm{Pi}=$ $1,000 \mathrm{~J} 2$ ). Resistance or susceptibility of $S$. sisymbriifolium was measured using the nematode multiplication rate, with values of $\mathrm{Pf} / \mathrm{Pi}<0.1=$ highly resistant, $\mathrm{Pf} / \mathrm{Pi} \leq 1=$ resistant, $\mathrm{Pf} / \mathrm{Pi} \geq 1=$ susceptible, and $\mathrm{Pf} / \mathrm{Pi} \geq 10=$ highly susceptible (Hajihassani et al. 2019b).

Reproduction of $M$. enterolobii in the greenhouse. Because M. enterolobii has not been found in Georgia, the host screening tests were conducted in North Carolina and South Carolina using three isolates of the nematode. Seedlings of S. sisymbriifolium and tomato (susceptible control) were grown as described previously and transplanted individually into 4-in.-deep plastic pots. The plants were inoculated with 10,000 eggs suspended in $1 \mathrm{ml}$ of water and placed on the greenhouse bench in a completely randomized design, with six replicates for each cultivar tested.

Eight weeks after nematode infection, the root systems were visually scored for percent galling based on a scale of 0 to 100 , with 0 representing no galls and 100 representing severe (100\%) galling (Bridge and Page 1980). Egg extraction from infected roots and assessment of $M$. enterolobii reproduction was performed as described above.

Histopathological study. Histological examinations were performed to evaluate the S. sisymbriifolium response to Meloidogyne spp. infection. Three to five segments ( 1 to $2 \mathrm{~cm}$ long) of galled (knotted) root from each infected plant were randomly selected for examinations. Root segments, including individual small knots and adjoining portions of unknotted tissue, were fixed in buffered formaldehyde solution for 24 to $48 \mathrm{~h}$ at room temperature. The fixed segments were dehydrated in a graded ethanol series (70 to $100 \%$ ) using a tissue processor (Excelsior AS; Thermo Scientific, Thermo Shandon Limited, Runcorn, Cheshire, U.K.) and embedded in paraffin. Cross-sections (4 to $5 \mu \mathrm{m}$ thick) were prepared using a rotary microtome (RM2125 RTS; Leica, Leica Biosystems, Nussloch, Germany) equipped with a knife, then mounted on glass slides. The sections were stained with hematoxylin-eosin, examined microscopically (Olympus BX43; Olympus, Tokyo, Japan), and photographed using a digital camera (Olympus DP73; Olympus).

Reproduction of $M$. arenaria, $M$. incognita, and M. haplanaria in microplots. A microplot study was set up in spring 2018 and 2019 to investigate whether the characterized resistance in $S$. sisymbriifolium to $M$. arenaria race $1, M$. incognita race 3 , and $M$. haplanaria was effective in the soil with high infestation pressure under field conditions. Experiments were conducted in cylindrical ( $76 \mathrm{~cm}$ in diameter), bottomless stainless steel drums extending $53 \mathrm{~cm}$ below and $10 \mathrm{~cm}$ above the soil surface and filled with a sandy loam soil ( $86 \%$ sand, $7 \%$ clay, and $7 \%$ silt) located at the University of Georgia, Tifton Campus, GA. Microplots were fumigated with metam sodium (AMVAC Chemical Corporation) approximately 4 weeks prior to initiation of experiments. Each microplot was soil drenched with $100 \mathrm{ml}$ of metam sodium mixed with 30 liter of tap water (Hajihassani et al. 2019a). Seedlings of S. sisymbriffolium line Sis Syn II and susceptible tomato (Rutgers) were prepared as stated previously and transplanted individually into the center of each microplot approximately $2 \mathrm{~cm}$ deeper than the soil surface on 21 April 2018 in the first trial and on 10 May 2019 in the repeat trial. A suspension of 6,000 J2/plant for each of $M$. arenaria, M. incognita, or $M$. haplanaria was pipetted into four 5 -cm-deep holes at the base of the plants. A completely randomized design with four replications per treatment were used. Microplots were irrigated uniformly every day and fertilized once postplanting with NPK (10-10-10) at $9.0 \mathrm{~kg} / \mathrm{ha}$. Daily air temperatures were collected from the Coastal Plain Experiment Station weather station (http:// www.georgiaweather.net), Tifton, GA. Sixty days after nematode inoculation, roots of individual plants were thoroughly washed free of soil, weighed, examined for gall severity, and processed to extract eggs as described above. The number of the eggs was determined using a light microscope and the multiplication rate of the nematodes was determined as specified previously. Based on the

TABLE 2. Reproduction of Meloidogyne enterolobii isolates from North Carolina (NC1 and NCSP) and South Carolina (BASP) on Solanum sisymbriifolium in the greenhouse ${ }^{\mathrm{w}}$

\begin{tabular}{|c|c|c|c|c|}
\hline Cultivars & Root fresh weight (g) & Percent root galling ${ }^{\mathrm{x}}$ & Number of eggs/g of fresh root & Multiplication rate \\
\hline \multicolumn{5}{|l|}{$\mathrm{NC} 1$} \\
\hline Rutgers $^{z}$ & $30.5 \pm 2.4 \mathrm{a}$ & $25.0 \pm 2.1 \mathrm{a}$ & $1,692.2 \pm 399.0 \mathrm{a}$ & $6.05 \pm 1.290 \mathrm{a}$ \\
\hline Diamond & $17.4 \pm 2.6 \mathrm{bc}$ & $5.3 \pm 0.7 \mathrm{~b}$ & $7.5 \pm 0.8 \mathrm{~b}$ & $0.01 \pm 0.001 \mathrm{~b}$ \\
\hline Sis Syn II & $14.3 \pm 2.5 \mathrm{c}$ & $4.1 \pm 0.6 \mathrm{~b}$ & $16.6 \pm 3.1 \mathrm{~b}$ & $0.02 \pm 0.002 \mathrm{~b}$ \\
\hline \multicolumn{5}{|l|}{ NCSP } \\
\hline Rutgers & $4.3 \pm 0.8 \mathrm{a}$ & $83.0 \pm 7.2 \mathrm{a}$ & $5,642.7 \pm 850.8 \mathrm{a}$ & $2.08 \pm 0.4 \mathrm{a}$ \\
\hline Diamond & $4.8 \pm 1.3 \mathrm{a}$ & $21.2 \pm 14.5 \mathrm{~b}$ & $2,989.3 \pm 2,670.3 \mathrm{a}$ & $0.64 \pm 0.1 \mathrm{~b}$ \\
\hline \multicolumn{5}{|l|}{ BASP } \\
\hline Rutgers & $13.0 \pm 1.6 \mathrm{a}$ & $86.4 \pm 3.2 \mathrm{a}$ & $6,728.0 \pm 1,335.1 \mathrm{a}$ & $7.66 \pm 0.8 \mathrm{a}$ \\
\hline Diamond & $5.4 \pm 0.9 \mathrm{a}$ & $9.9 \pm 1.7 \mathrm{c}$ & $0.0 \pm 0.0 \mathrm{~b}$ & $0.01 \pm 0.0 \mathrm{~b}$ \\
\hline White Star & $5.6 \pm 1.2 \mathrm{a}$ & $18.3 \pm 5.7 \mathrm{bc}$ & $3,001.8 \pm 2571.1 \mathrm{a}$ & $0.36 \pm 0.3 b$ \\
\hline Sis Syn II & $5.1 \pm 0.7 \mathrm{a}$ & $25.7 \pm 2.0 \mathrm{~b}$ & $9.1 \pm 8.0 \mathrm{~b}$ & $0.01 \pm 0.0 \mathrm{~b}$ \\
\hline
\end{tabular}

${ }^{w}$ Each value signifies the mean \pm standard error $(n=12)$ of each treatment. Means followed by the same letter within columns are not significantly different $(P=0.05)$ based on Tukey's test.

${ }^{x}$ Rated on a scale of 0 to 100 , with 0 representing no galls and 100 representing severe (100\%) root galling.

y Multiplication rate $=$ the number of eggs on roots at harvest/inoculated juveniles at planting.

${ }^{\mathrm{z}}$ Rutgers = susceptible tomato control. Each plant was inoculated with 10,000 eggs of each nematode species at transplanting. 
multiplication rate, the cultivars were classified as resistant or susceptible.

Data analysis. The root galling, egg count, and multiplication rate in both greenhouse and microplot studies were subjected to a one-way analysis of variance (ANOVA) using PROC Mixed within SAS (v. 9.4; SAS Institute, Cary, NC, U.S.A.). Prior to ANOVA, data were combined for each nematode species because the initial and repeat experiments did not differ. Plant variety was considered a fixed effect and trial/year was a random effect. Means values were compared using Tukey's adjustment for multiple comparison test at $P=0.05$. Means were separated with Tukey's adjustment for multiple comparisons test. Least-squares means $t$ tests demonstrating if the mean was significantly different from zero were used to determine whether the nematode multiplication rate was different
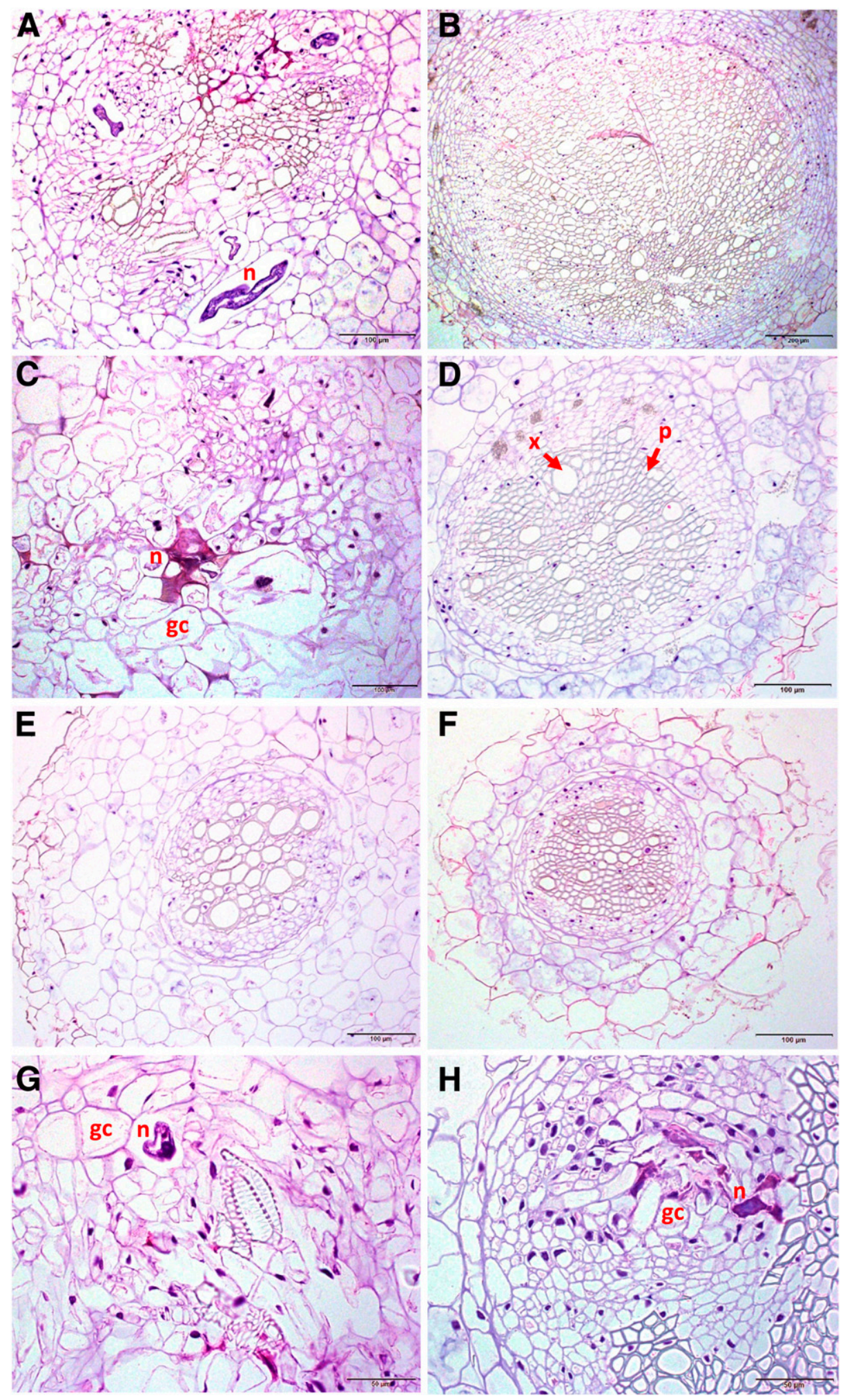

Fig. 1. Hematoxylin-eosin-stained cross-sections of Solanum sisymbriifolium root tissues infected with Meloidogyne arenaria in A, Diamond and B, Sis Syn II; M. incognita in C, Diamond and D, Sis Syn II; M. haplanaria in E, Diamond and F, Sis Syn II; and M. javanica in G, Diamond and $\mathbf{H}$, SIS Syn II 8 weeks after inoculation. $\mathrm{n}=$ adult female nematode and $\mathrm{gc}=$ giant cell. Arrows indicate xylem $(\mathrm{x})$ and phloem $(\mathrm{p})$ elements. 

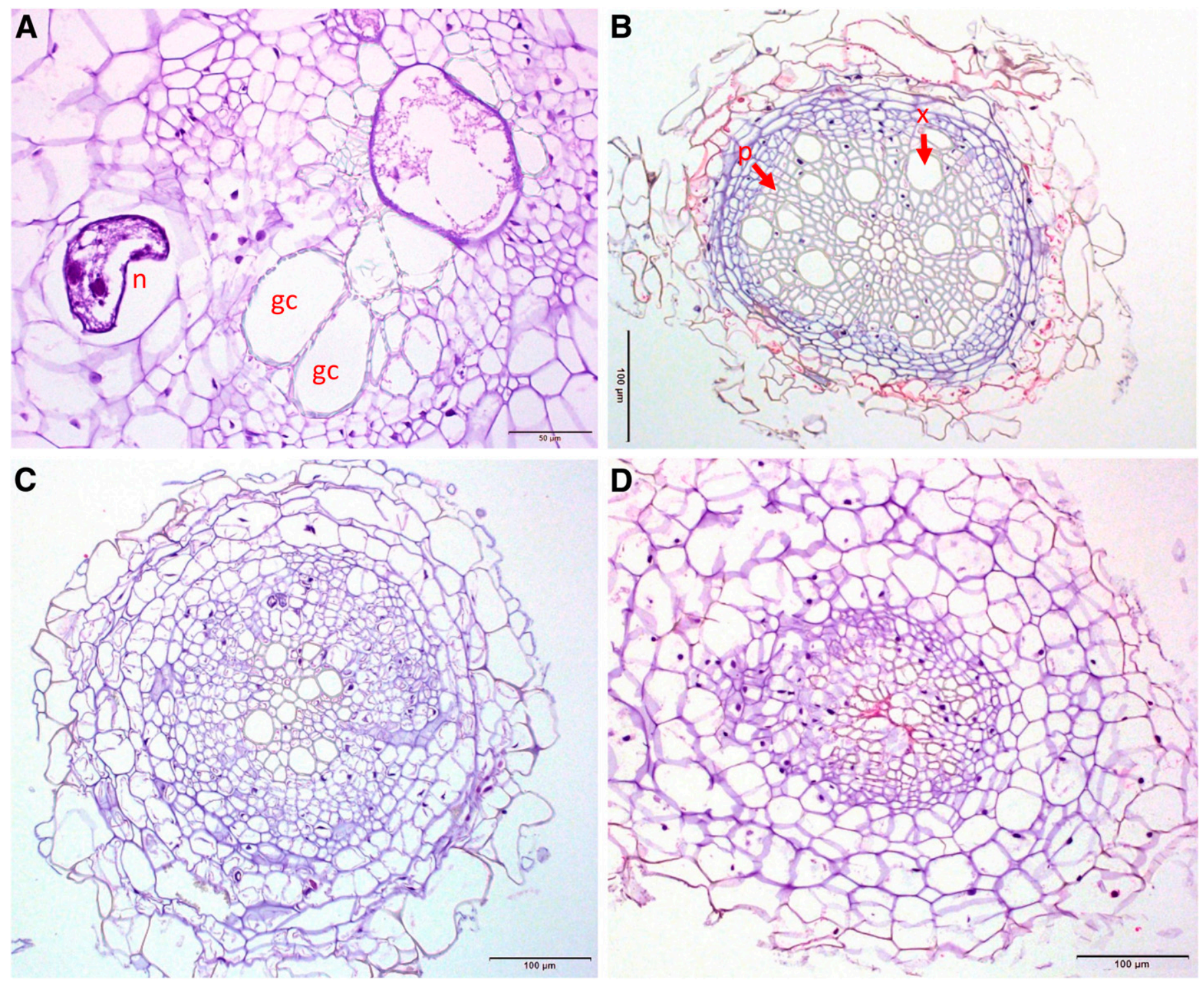

Fig. 2. Susceptibility and resistance responses in the root systems of tomato and Solanum sisymbriifolium infected with Meloidogyne enterolobii isolate NCSP 8 weeks after inoculation. Cross-sections of root tissues of A, susceptible Rutgers tomato and resistant S. sisymbriifolium B, Diamond; C, White Star; and D, Sis Syn II stained with hematoxylin-eosin. $\mathrm{n}=$ adult female nematode and $\mathrm{gc}=$ giant cell. Arrows indicate xylem (x) and phloem (p) elements.

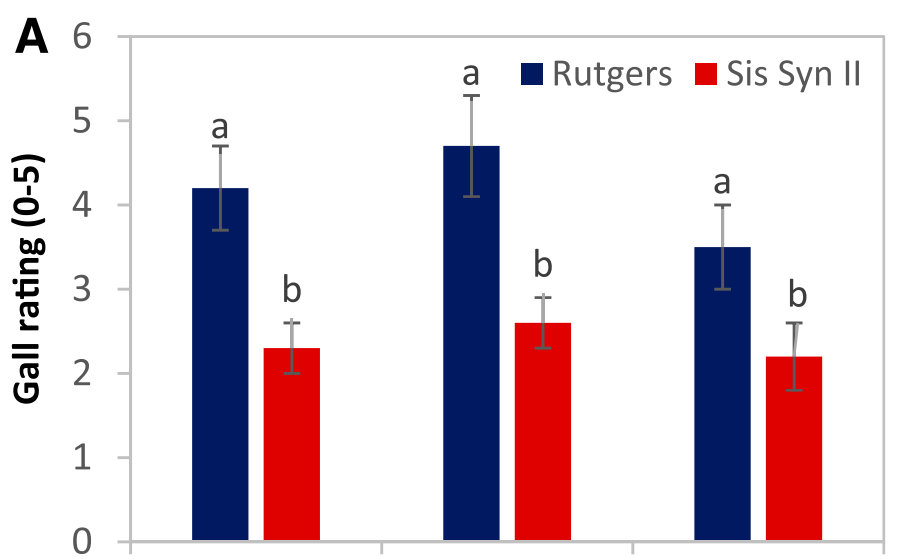

M. arenaria M. incognita M. haplanaria
M. arenario

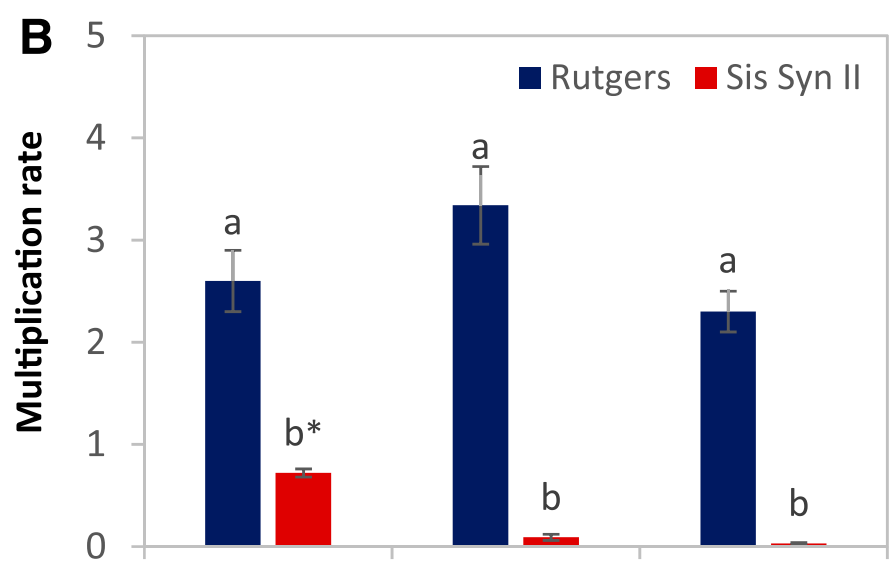

M. incognita M. haplanaria

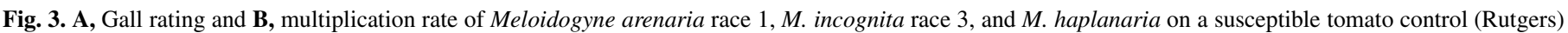

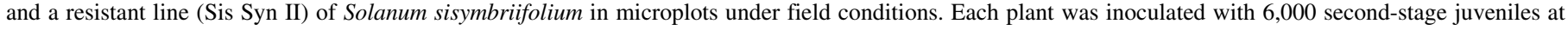

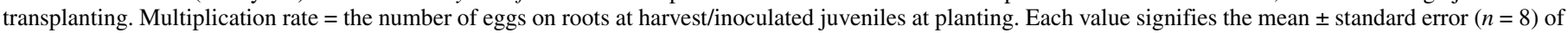

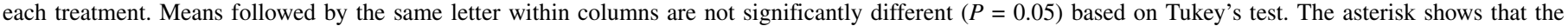
multiplication rate is not different from 1 according to least-squares means $t$ tests. 
from 1 (the base 10 algorithm of 1 is 0 ). Therefore, single $t$ tests that showed that the treatment mean was significantly different from 0 indicated that the multiplication rate deviated significantly from 1 (Hajihassani et al. 2016).

\section{RESULTS}

Reproduction of M. arenaria, M. incognita, M. haplanaria, and $M$. javanica in the greenhouse. Similar to the susceptible tomato control, all S. sisymbriifolium cultivars or lines produced visible root galling in response to $M$. arenaria, $M$. incognita, and $M$. haplanaria infection; however, no significant difference among the cultivars was observed. Compared with the tomato control, however, Sis Syn II had a lower $(P \leq 0.0001)$ gall rating on $S$. sisymbriifolium infected with either $M$. arenaria or $M$. incognita (Table 1). All S. sisymbriifolium cultivars infected with $M$. javanica had root galling in the range of 2.6 to 3.3, with no difference among them (Table 1). Sis Syn II had the least number of $M$. arenaria eggs per gram of fresh root weight as compared with the tomato control. No significant difference for the $M$. arenaria egg counts was observed among Quattro, Diamond, and White Star. The number of $M$. incognita and $M$. haplanaria eggs per gram of root was greater $(P \leq 0.0001)$ in tomato than in all $S$. sisymbriifolium cultivars examined; however, no difference among the cultivars was observed. No significant difference for the M. javanica egg counts was observed among tomato control and S. sisymbriifolium cultivars. The multiplication rate of $M$. arenaria for Sis Syn II was 0.4 (Table 1), indicating resistance. $M$. incognita failed to reproduce on Quattro, White Star, and Sis Syn II, having a multiplication rate of $0.2,0.12$, and 0.07 , respectively. All $S$. sisymbriifolium cultivars sustained the lowest multiplication rate of $M$. haplanaria when compared with the tomato control $(P \leq$ $0.001)$. The multiplication rate of $M$. javanica ranged from 10 to 20 , showing that the nematode was highly pathogenic on all S. sisymbriifolium lines (Table 1).

All three isolates of M. enterolobii from North Carolina and South Carolina induced root galling on S. sisymbriifolium. The percent root galling, varying from 3.5 to 25.7 for all $S$. sisymbriifolium cultivars, was significantly lower $(P \leq 0.0001)$ than that of the tomato control (Table 2 ). The root galling did not often vary among the cultivars tested. No significant difference was observed among Diamond, White Star, and Sis Syn II in egg production when inoculated with the NC1 isolate of $M$. enterolobii. In contrast, Diamond infected with the NCSP isolate had greater numbers of egg per gram of root compared with White Star and Sis Syn II. For the BASP isolate, White Star had higher egg counts in comparison with Diamond and Sis Syn II. The multiplication rates of M. enterolobii isolates from North Carolina and South Carolina for all S. sisymbriifolium cultivars varied from 0.01 to 0.64 , with no difference among the cultivars (Table 2).

Histopathological study. Histological observations of galled root tissues indicated differences in resistance or susceptibility among $S$. sisymbriifolium lines. Root tissues of the susceptible tomato control infected with $M$. arenaria, $M$. incognita, $M$. haplanaria, or $M$. javanica had multiple giant cells 8 weeks after nematode inoculation (not shown). Cross-sections of the root system of Meloidogyne-infected S. sisymbriifolium revealed morphological differences between susceptible Diamond and resistant Sis Syn II cultivars. Multiple giant cells were observed in the root tissues of Diamond infected with M. arenaria (Fig. 1A), M. incognita (Fig. 1C), or M. javanica (Fig. 1G). No sign of feeding site and giant cell development was detected in the root tissues of Diamond infected with M. haplanaria (Fig. 2E) and Sis Syn II infected with $M$. arenaria (Fig. 1B), M. incognita (Fig. 1D), or $M$. haplanaria (Fig. 1F). In contrast, degraded giant cells were observed in the root tissues of Sis Syn II infected with $M$. javanica. The root tissues of Diamond infected with M. arenaria (Fig. 2A), M. incognita (Fig. 2C), and M. javanica (Fig. 2G) showed severe hypertrophy. Limited hypertrophy was observed in the vascular cylinder and the cortical cells of Sis Syn II infected with $M$. arenaria (Fig. 2B), M. incognita (Fig. 2D), or M. haplanaria (Fig. 2F), suggesting that the $\mathrm{J} 2$ entered the roots but failed to establish a feeding site.

Cross-sections of the root system of $M$. enterolobii-infected Rutgers tomato revealed visible giant cells and nematode females (Fig. 2A). No feeding site formation was detected in the root tissues of Sis Syn II (Fig. 2C and D) and other lines or cultivars (not shown) inoculated with $M$. enterolobii.

Reproduction of $M$. arenaria, $M$. incognita, and $M$. haplanaria in microplots. The average daily air temperature during the experiments was $24.0^{\circ} \mathrm{C}$ (ranging from 9.9 to $35.2^{\circ} \mathrm{C}$ ) in 2018 and $26.8^{\circ} \mathrm{C}\left(12.8\right.$ to $\left.37.3^{\circ} \mathrm{C}\right)$ in 2019 . Root galling on the S. sisymbriifolium line Sis Syn II infected with $M$. incognita, $M$. incognita, or $M$. haplanaria was lower $(P \leq 0.001)$ than that of
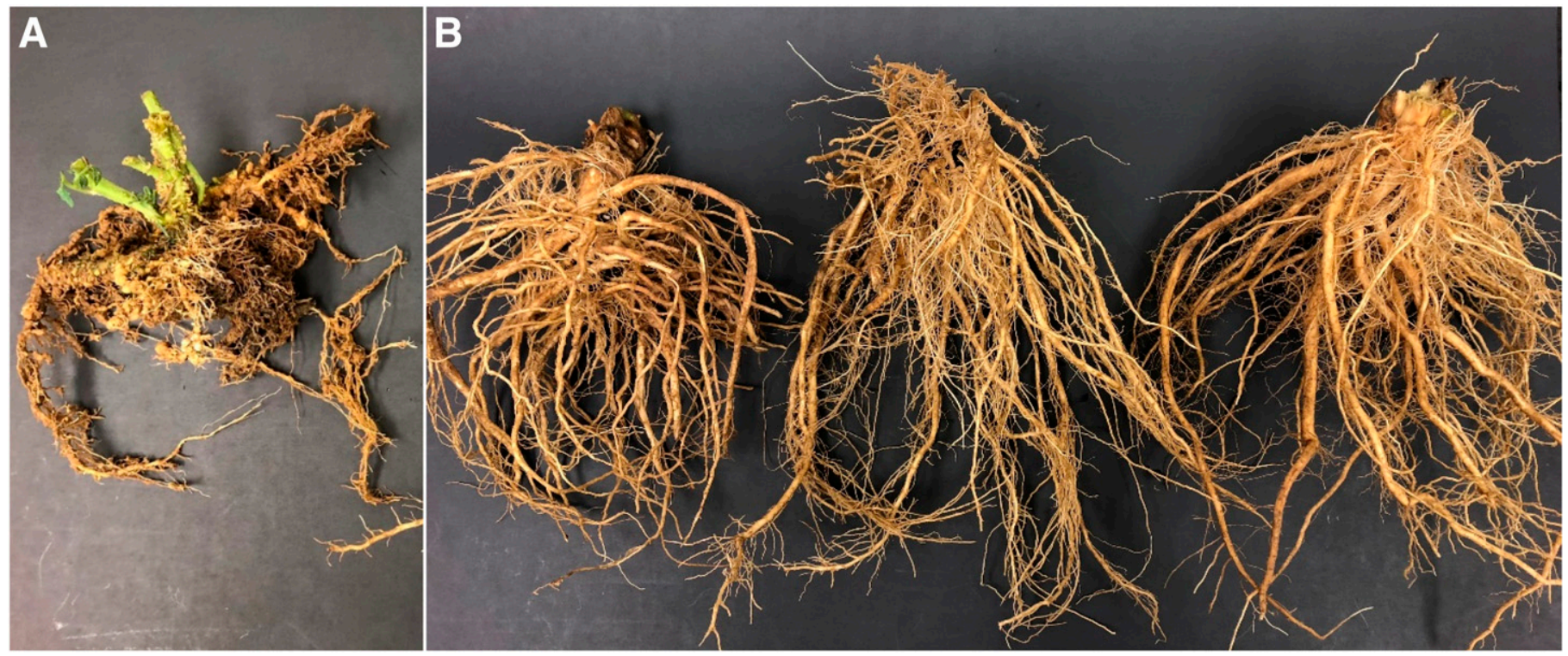

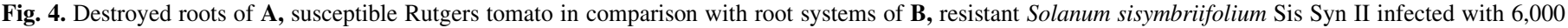
second-stage juveniles of Meloidogyne arenaria (left), M. incognita (middle), and M. haplanaria (right) in microplots. 
the tomato control (Figs. 3A and 4). Sis Syn II had root galling values $>2$ (Fig. 3A) but had lower egg production per gram of root compared with the tomato control (data not shown). The multiplication rate of $M$. arenaria, $M$. incognita, and $M$. haplanaria on S. sisymbriifolium was significantly greater than that of the susceptible tomato (Fig. 3B).

\section{DISCUSSION}

We documented the presence of resistance to $M$. arenaria race $1, M$. incognita race $3, M$. haplanaria, and $M$. enterolobii in different $S$. sisymbriifolium cultivars or lines. Resistance to parasitic nematodes is characterized as the ability of a plant species to prevent nematode development or reproduction. Various factors for determining the resistance of plants to Meloidogyne spp. have been used previously. The most common procedures are the number or percentage of galls or egg masses produced as well as the Pf/Pi values (Sasser et al. 1984). All Meloidogyne spp. tested in the present study induced root galling (ranging from 1.9 to 3.7 ) with minimal to moderate egg production in the roots of resistant $\mathrm{S}$. sisymbriifolium genotypes. This may show that, although galls were formed, the nematodes failed to reach maturity and complete their life cycle on $\mathrm{S}$. sisymbriifolium. Our histological examinations also revealed a typical reaction of susceptible tomato and $S$. sisymbriifolium cultivars to all Meloidogyne spp., with multiple feeding sites and limited giant cell modifications, as described previously in the literature for the root-knot nematodes (Abad et al. 2003; Cabasan et al. 2014). In contrast, the root structures of resistant genotypes of $S$. sisymbriifolium were healthy.

In this study, the susceptibility or resistance of S. sisymbriifolium for Meloidogyne spp. was determined by comparing the nematode multiplication rate $(\mathrm{Pf} / \mathrm{Pi})$ of each genotype to the tomato control. The effectiveness of resistance to $M$. arenaria varied greatly between $S$. sisymbriifolium lines. Quattro and White Star exhibited susceptibility to the nematode and Diamond was the most susceptible genotype, with a multiplication rate of 11.7. Resistance to $M$. arenaria was observed only in Sis Syn II in both greenhouse and microplot studies; however, the reproduction of $M$. arenaria on Sis Syn II in the microplot study was numerically greater than that in the greenhouse test. The increased reproduction of Sis Syn II in the microplot test was probably due to the higher temperature $\left(>30^{\circ} \mathrm{C}\right)$ on numerous days in the field in 2018 and 2019 compared with the fluctuating ambient temperatures in the greenhouse.

The level of resistance in $S$. sisymbriifolium to $M$. incognita varied from highly resistant (Sis Syn II) to resistant (Quattro and White Star) to susceptible (Diamond). In the microplots, Sis Syn II had $95 \%$ fewer eggs per gram of fresh root weight than the tomato control, indicating that this genotype was highly resistant to $M$. incognita. The evaluation of resistance of $S$. sisymbriifolium at fluctuating soil temperatures under field conditions, before its use as a rootstock for grafting tomato, will help to develop better management strategies for root-knot nematodes. We found that $M$. javanica aggressively reproduced in the roots, indicating that no resistance to this nematode was present in all $S$. sisymbriifolium cultivars tested. In a study conducted in Portugal, the S. sisymbriifolium cultivar Domino was found to be resistant to $M$. javanica (Dias et al. 2012).

In both greenhouse and microplot tests, S. sisymbriifolium revealed medium to high levels of resistance to $M$. haplanaria, with a multiplication rate $<1$ compared with the susceptible tomato. Similarly, $S$. sisymbriifolium was found to be highly resistant to multiple M. enterolobii isolates, with the multiplication rate $\leq 0.03$. However, we noticed that only 1 of 12 plants from White Star and Diamond cultivars failed to prevent $M$. enterolobii reproduction, resulting in an increased multiplication rate of 0.64 and 0.36 for White Star and Diamond, respectively. This variation in M. enterolobii reproduction on White Star and Diamond could be attributed to segregation of a resistance gene or genetic variability in the seed population of $S$. sisymbriifolium. Both $M$. haplanaria and $M$. enterolobii are capable of breaking the resistance $M i$ gene in tomato (Brito et al. 2004; Kiewnick et al. 2008). Because no cultivar or line of $S$. sisymbriffolium exhibited susceptibility to M. enterolobii and M. haplanaria, it is possible that the resistance to these two species may be based on the same resistance gene or genes. This will need to be further investigated with genetic studies.

The presence of resistance in S. sisymbriifolium to plantparasitic nematodes, as previously reported for Globodera pallida, G. rostochiensis, M. chitwoodi, and M. javanica (Dias et al. 2012; Scholte and Vos 2000), has been confirmed in this work for other major species of root-knot nematodes. It has been reported that growing S. sisymbriffolium in rotation with potato did not lead to an increase in the egg production of G. pallida in soil when compared with a susceptible potato cultivar (Dias et al. 2017; Scholte and Vos 2000). Similarly, S. sisymbriifolium exhibited resistance to $M$. incognita and, consequently, has been used as a rootstock for tomato production in some areas in Asia (Baidya et al. 2017; Matsuzoe et al. 1993).

Tomato production in the United States relies extensively on soil fumigation for managing root-knot nematodes and other pathogens; however, fumigants face regulatory restrictions to protect the environment and human health. The ability of some Meloidogyne spp. or isolates to overcome the $M i-1$ resistance can restrict the use of resistant tomato cultivars for successful management of the nematode. Using $S$. sisymbriifolium as a resistant rootstock could result in an effective control through reducing the population density of Meloidogyne spp. in the soil in an integrated management program. This management option will provide the opportunity for growers to use more diversified crop rotations and enhance economical crop production. Future studies on the efficacy of $S$. sisymbriifolium cultivars as resistant rootstocks, when grafted with scions of susceptible tomato, will be investigated in field trials to evaluate their level of resistance and ability to enhance tomato yield in the presence of Meloidogyne spp.

\section{ACKNOWLEDGMENTS}

We thank C. Brown (United States Department of AgricultureAgricultural Research Service, Prosser, WA, U.S.A.) for supplying the seed of S. sisymbriifolium line Sis Syn II.

\section{LITERATURE CITED}

Abad, P., Favery, B., Rosso, M. N., and Castagnone-Sereno, P. 2003. Root-knot nematode parasitism and host response: Molecular basis of a sophisticated interaction. Mol. Plant Pathol. 4:217-224.

Abdul-Baki, A. A., Haroon, S. A., and Chitwood, D. J. 1996. Temperature effects on resistance to Meloidogyne spp. in excised tomato roots. HortScience 31:147-149.

Baidya, S., Timila, R. D., Bahadur, K. C. R., Manandhar, H. K., and Manandhar, C. 2017. Management of root knot nematode on tomato through grafting rootstock of Solanum sisymbriifolium. J. Nepal Agric. Res. Counc. 3:27-31.

Bailey, D. M. 1941. The seedling method for root-knot nematode resistance. Proc. Am. Soc. Hortic. Sci. 38:573-575.

Baker, K. R., Shoemaker, P. B., and Nelson, L. A. 1976. Relationship of initial population densities of Meloidogyne incognita and M. hapla to yield of tomato. J. Nematol. 8:232-239.

Bridge, J., and Page, S. L. J. 1980. Estimation of root-knot nematode infestation levels on roots using a rating chart. Trop. Pest Manage. 26: 296-298.

Brito, J. A., Powers, T. O., Mullin, P. G., Inserra, R. N., and Dickson, D. W. 2004. Morphological and molecular characterization of Meloidogyne mayaguensis isolates from Florida. J. Nematol. 36:232-240.

Cabasan, M. T. N., Umar, A., Bellafiore, S., and De Waele, D. 2014. Histopathology of the rice root-knot nematode, Meloidogyne graminicola, on Oryza sativa and O. glaberrima. Nematology 16:73-81.

Castagnone-Sereno, P., Bongiovanni, M., and Dalmasso, A. 1994. Reproduction of virulent isolates of Meloidogyne incognita on susceptible and Mi-resistant tomato. J. Nematol. 26:324-328. 
Church, G. T. 2005. First report of the root-knot nematode Meloidogyne floridensis on tomato (Lycopersicon esculentum) in Florida. Plant Dis. 89:527.

de Carvalho, L. M., Benda, N. D., Vaughan, M. M., Cabrera, A. R., Hung, K., Cox, T., Abdo, Z., Allen, L. H., and Teal, P. E. A. 2015. Mi-1-mediated nematode resistance in tomatoes is broken by short-term heat stress but recovers over time. J. Nematol. 47:133-140.

Dias, M. C., Conceição, I. L., Abrantes, I., and da Cunha, M. J. M. 2012. Solanum sisymbriifolium-A new approach for the management of plantparasitic nematodes. Eur. J. Plant Pathol. 133:171-179.

Dias, M. C., Perpétuo, L. S., Cabral, A. T., Guilherme, R., da Cunha, M. J. M., Melo, F., Machado, Ó. C., and Conceição, I. L. 2017. Effects of Solanum sisymbriifolium on potato cyst nematode populations in Portugal. Plant Soil 421:439-452.

Dropkin, V. H. 1969. Necrotic reaction of tomatoes and other hosts resistant to Meloidogyne: Reversal by temperature. Phytopathology 59:1632-1637.

Gilbert, J. C., and McGuire, D. C. 1956. Inheritance of resistance to severe root-knot from Meloidogyne incognita in commercial type tomatoes. Proc. Am. Soc. Hortic. Sci. 68:437-442.

Hajihassani, A. 2018. Chemical nematicides for control of plant-parasitic nematodes in Georgia vegetable crops. University of Georgia, College of Agriculture and Environmental Sciences Cooperative Extension Service Bulletin 1502. https://secure.caes.uga.edu/extension/publications/files/pdf/ B\%201502_1.PDF

Hajihassani, A., Davis, R. F., and Timper, P. 2019a. Evaluation of selected nonfumigant nematicides on increasing inoculation density of Meloidogyne incognita on cucumber. Plant Dis. 103:3161-3165.

Hajihassani, A., Rutter, W. B., and Luo, X. 2019b. Resistant pepper carrying $\mathrm{N}, \mathrm{Mel}$, and $\mathrm{Me} 3$ have different effects on penetration and reproduction of four major Meloidogyne species. J. Nematol. 51:1-9.

Hajihassani, A., Tenuta, M., and Gulden, R. H. 2016. Host preference and seed-borne transmission of the stem nematodes, Ditylenchus weischeri and D. dipsaci on select pulse and non-pulse crops grown in the Canadian Prairies. Plant Dis. 100:1087-1092.

Hussey, R. S. 1985. Host-parasite relationships and associated physiological changes. Pages 143-153 in: An Advanced Treatise on Meloidogyne. J. N. Sasser and C. C. Carter, eds. Vol. I. Biology and Control. North Carolina State University Graphics, Raleigh, NC, U.S.A.

Hussey, R. S., and Barker, K. R. 1973. Comparison of methods of collecting inocula of Meloidogyne spp., including a new technique. Plant Dis. Rep. 57: 1025-1028.

Hussey, R. S., and Janssen, G. J. W. 2002. Root-knot nematodes: Meloidogyne species. Pages 43-70 in: Plant Resistance to Parasitic Nematodes. J. L. Starr, R. Cook, and J. Bridge, eds. CABI Publishing, Wallingford, U.K.

Iberkleid, I., Ozalvo, R., Feldman, L., Elbaz, M., Bucki, P., and Horowitz, S. B. 2014. Responses of tomato genotypes to avirulent and Mi-virulent Meloidogyne javanica isolates occurring in Israel. Phytopathology 104:484-496.

Joseph, S., Mekete, T., Danquah, W. B., and Noling, J. 2016. First report of Meloidogyne haplanaria infecting Mi-resistant tomato plants in Florida and its molecular diagnosis based on mitochondrial haplotype. Plant Dis. 100: 1438-1445.

Kartesz, J. T. 2012. North American Plant Atlas [maps generated from Kartesz, J. T. 2010. Floristic Synthesis of North America, Version 1.0. Biota of North America Program (BONAP)]. The Biota of North America Program (BONAP), Chapel Hill, NC, U.S.A. http://www.bonap.org/MapSwitchboard.html

Kiewnick, S., Karssen, G., Brito, J. A., Oggenfuss, M., and Frey, J. E. 2008. First report of root-knot nematode Meloidogyne enterolobii on tomato and cucumber in Switzerland. Plant Dis. 92:1370.

Marquez, J., Ye, W., Skantar, A., and Hajihassani, A. 2019. Molecular identification of Meloidogyne spp. associated with vegetable crops in southern Georgia. In: Proc. 58th Annu. Meet. Soc. Nematol. Raleigh, NC, U.S.A.

Matsuzoe, N., Okubo, H., and Fujieda, K. 1993. Resistance of tomato plants grafted on Solanum rootstocks to bacterial wilt and root-knot nematode. J. Jpn. Soc. Hortic. Sci. 61:865-872.

Ornat, C., Verdejo-Lucas, S., and Sorribas, F. J. 2001. A population of Meloidogyne javanica from Spain virulent to the $M i$-resistance gene in tomato. Plant Dis. 85:271-276.

Rutter, W. B., Skantar, A. M., Handoo, Z. A., Mueller, J. D., Aultman, S. P., and Agudelo, P. 2019. Meloidogyne enterolobii found infecting root-knot nematode resistant sweetpotato in South Carolina, United States. Plant Dis. 103:775.

Sasser, J., Eisenback, J., Carter, C., and Triantaphyllou, A. 1983. The international Meloidogyne project: Its goals and accomplishments. Annu. Rev. Phytopathol. 21:271-288.

Sasser, J. N., Carter, C. C., and Hartman, K. M. 1984. Standardization of Host Suitability Studies and Reporting of Resistance to Root-Knot Nematodes. North Carolina State University Graphics, Raleigh, NC, U.S.A.

Scholte, K., and Vos, J. 2000. Effects of potential trap crops and planting date on soil infestation with potato cyst nematodes and root knot nematodes. Ann. Appl. Biol. 137:153-164.

Taylor, A. L., and Sasser, J. N. 1978. Biology, Identification, and Control of Root-Knot Nematodes (Meloidogyne species). North Carolina State University Graphics, Raleigh, NC, U.S.A.

Timmermans, B. G. H., Vos, J., Stomph, T. J., Van Nieuwburg, J., and Van Der Putten, P. E. L. 2006. Growth duration and root length density of Solanum sisymbriifolium (Lam.) as determinants of hatching of Globodera pallida (Stone). Ann. Appl. Biol. 148:213-222.

Tzortzakakis, E. A., Adam, M. A. M., Blok, V. C., Paraskevopoulos, C., and Bourtzis, K. 2005. Occurrence of resistance-breaking populations of rootknot nematodes on tomato in Greece. Eur. J. Plant Pathol. 113:101-105.

USDA-NASS. 2019. Vegetables 2018 Summary. United States Department of Agriculture-National Agricultural Statistics Service. https://usda.library. cornell.edu/concern/publications/02870v86p

Wesemael, W. M. L., Viaene, N., and Moens, M. 2011. Root-knot nematodes (Meloidogyne spp.) in Europe. Nematology 13:3-16.

Williamson, V. M. 1998. Root-knot nematode resistance genes in tomato and their potential for future use. Annu. Rev. Phytopathol. 36:277-293. 